\title{
Air Flow around a Rotating Pirn and Power Loss
}

\author{
Part 1: Experiment and Flow Analysis by a Finite Element Method
}

\author{
By Sukenori Shintaku, Juhachi Oda, Hiroyuki Yamazaki, Members TMSJ.
}

\author{
Faculty of Technology, Kanazawa University, Kanazawa
}

Based on the Journal of the Textile Machinery Society of Japan, Transactions, Vo.43, No.1, T1-T9(1990-1) and No.5, T34-T44(1990-5)

\begin{abstract}
Air flow around a pirn rotating at high speed and power loss to rotate it were measured, and analysed from the point of Reynolds number. Air is sucked from the upper and the lower parts of the pirn, and is blown out from its middle part. This phenomena is observed and analysed by using a finite element method. Flow velocity and power loss calculated from analysed results agree well with the measured. The energy loss can be reduced with a $20^{\circ}$ conical cover set around the pirn.
\end{abstract}

\section{Introduction}

In the processes of manufacturing yarns and aftertreatment, there are many kinds of machines to rotate yarn packages. The energy consumption in these machines presents a problem because those are run for a long hour, and the electric power cost occupies considerably a large part of running cost. For saving energy, power requirement should be analysed into several components, such as to drive pulleys and flat belts, to wind and take up yarns and to rotate spindles and packages so on. From this standpoint, the power to rotate the yarn package was treated in this paper. When a package is rotated, the air around it is sucked from the upper and the lower parts of the pirn and is blown out from the middle part. Thus the power to rotate the package is lost to stir the surrounding air. Accordingly, it is important to measure quantitatively the air flow. This flow was visualized by using the tuft method, and its velocity and the power to rotate the package were measured. The air velocity around the package and the energy loss were studied by a finite element method, and compared with experimental results.

\section{Air Flow around a Rotating Pirn}

As a typical package, the pirn shape was treated in this paper, which was $116 \mathrm{~mm}$ in diameter, $200 \mathrm{~mm}$ long and $420 \mathrm{~mm}$ high contained $20^{\circ}$ double-tapered parts. The yarn was nylon 70 denier, and wound by $3 \mathrm{~kg}$. The pirn surface seemed smooth, and in order to compare this surface, the pirn was over-wound with cotton yarn $30 \mathrm{~s}$ (19.6 tex). The air flow around the rotating pirn was visualized by the tuft method. Each tuft was composed of a small sponge piece and a yarn which was tied to a wire as shown in Fig.1. The yarn is stretched following the air flow, and the sponge piece visualizes the air flow without difficulty. Many tufts were set up around the rotating pirn, and pictures were taken. Figure

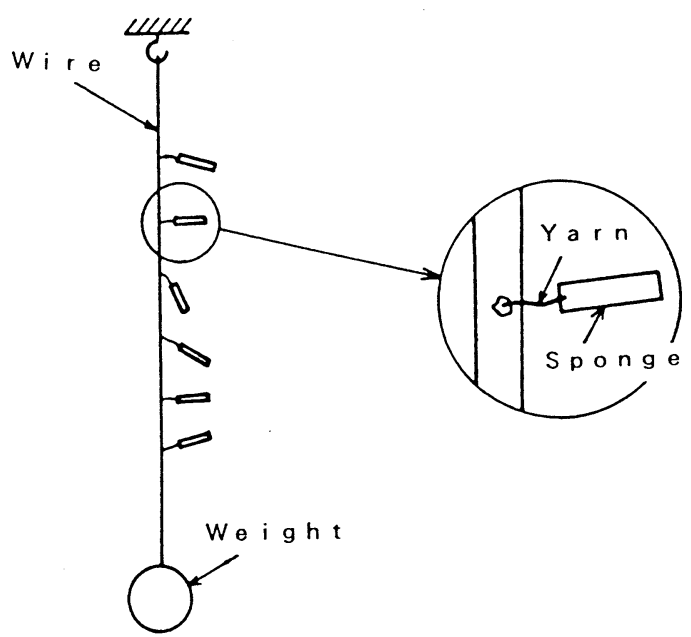

Fig. 1 Tuft consisted of yarn and sponge piece

2 shows the flow around the nylon-pirn rotating at 4,000rpm. Tufts in Fig.2(a) indicate the radial flow, and Fig.2(b) the circumferential flow around the rotating axis. The composition of these two gives the direction of true air-flow, but doesn't give the velocity. Fig.2(a), (b) show clearly that the air is blown out from the middle of the rotating pirn.

The apparatus used to measure the velocity and the power to rotate the pirn is shown schematically in Fig.3. The co-ordinate system is $r$ and $\theta$ in a plane normal to the rotating axis, and $z$ perpendicularly downward. The magnitude of the air-velocity was measured with a U-gauge. A sphere was set up at the end of a piano wire protruding from the U-gauge, and exposed to the stirring air to get the air force. This force $F_{b}$ was measured, and the air velocity was calculated by the following equation:

$$
F_{b}=C_{d} \cdot \rho \cdot V^{2} \cdot A / 2 \ldots \ldots \ldots \ldots
$$

where $A$ is the projected area of the sphere normal to the flow ( $10 \mathrm{~mm}$ in diameter), and $C_{d} 0.39$ by other experiment, $\rho$ the air density. The flow direction was known by visualization, and the variation of $V$ was less than 


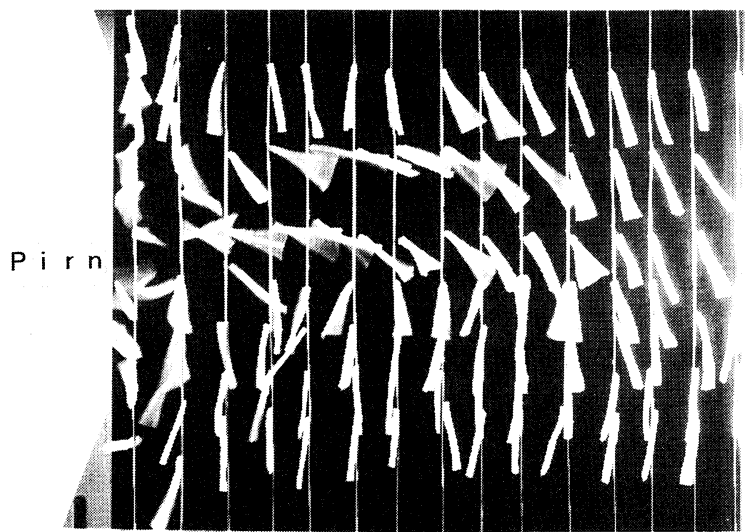

(a) A i Flow in $r-z$ P I a n e

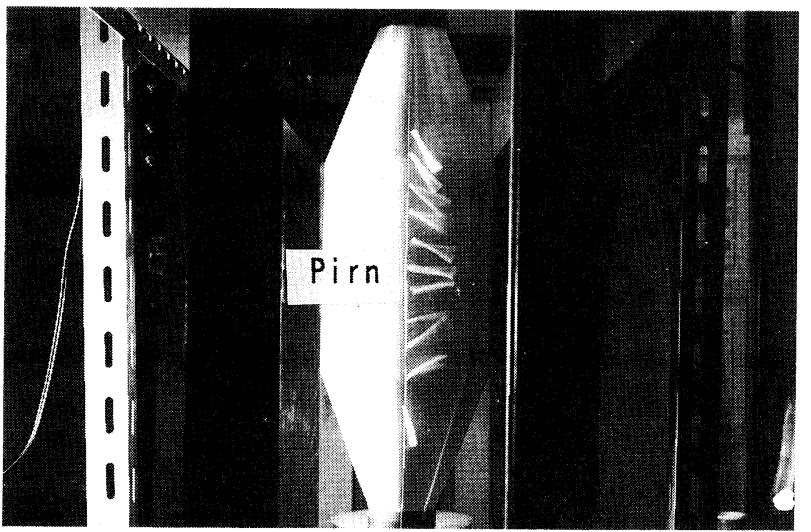

(b) A i Flow in $\theta-z$ PI a ne $(r=78 \mathrm{~mm})$

Fig. 2 Visualization of air flow around a rotating pirn by tufts
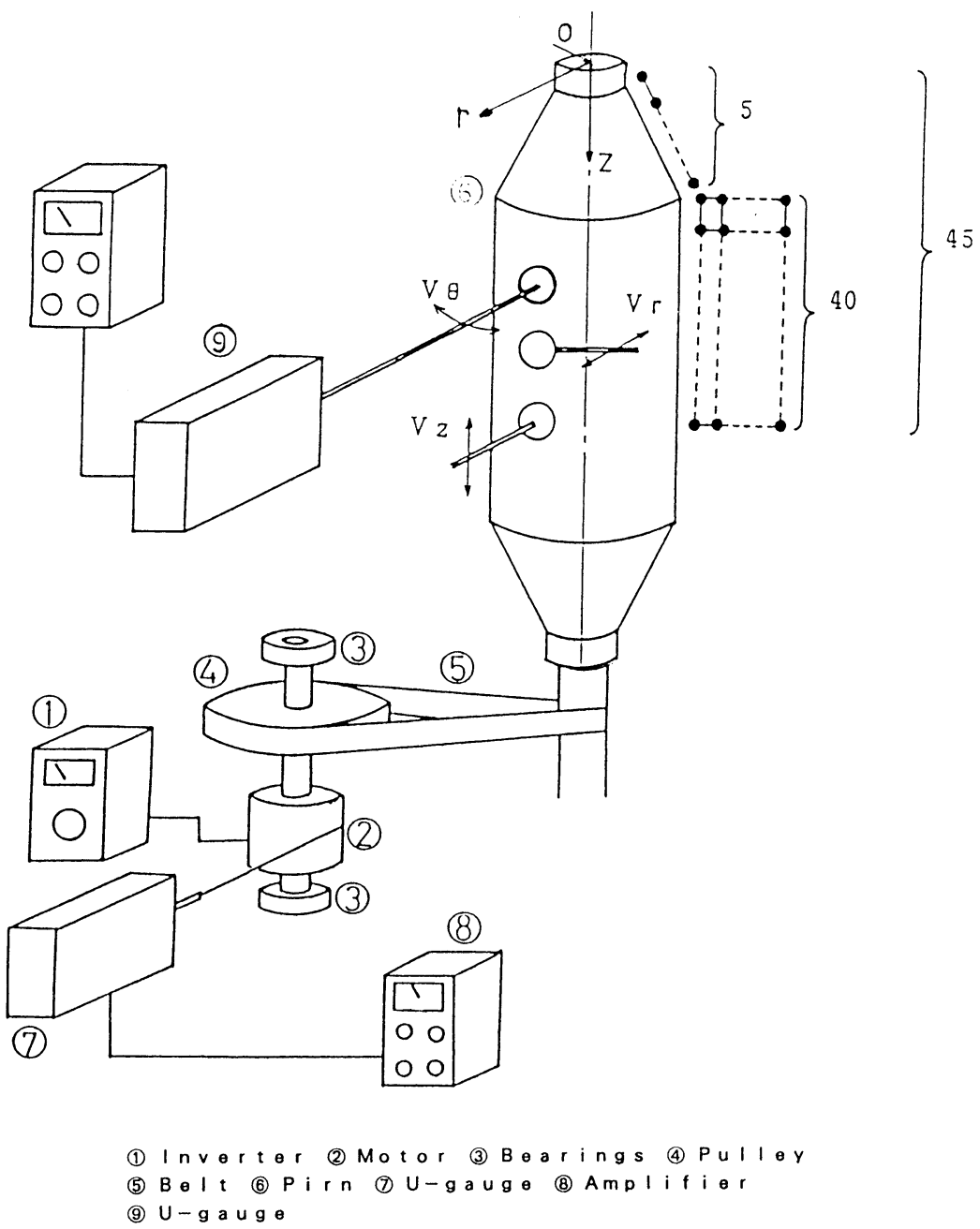

Fig. 3 Schematic of apparatus used to measure velocity and power

that of $F_{b}$ as $V$ is the square root of $F_{b}$. Velocity components of flow are shown in Fig. 4 and Fig. 5 plotted against the distance $z$ from the upper end of the pirn. Figure 4 shows the radial component $V_{r}$, the circumferential component $V_{\theta}$ and the axial component $V_{z}$ at points 68,88 and $108 \mathrm{~mm}$ apart from the rotating axis. Positive or negative sign of $V_{r}$ means blow-off or suction. That of $V_{z}$ means downward or upward flow. Figure 5 shows velocity components of the cotton and the nylon surfaces at the point $68 \mathrm{~mm}$ apart from the axis of rotation. $V_{r}$ indicates clear trend that the suction occurs at both ends and blow off at the middle, and that $V_{\theta}$ increases gradually towards the center, $V_{z}$ increasing slightly with increasing $Z$ with the reverse sign at the height center. Those trends do not have any relation to the condition of the pirn surface and the number of 


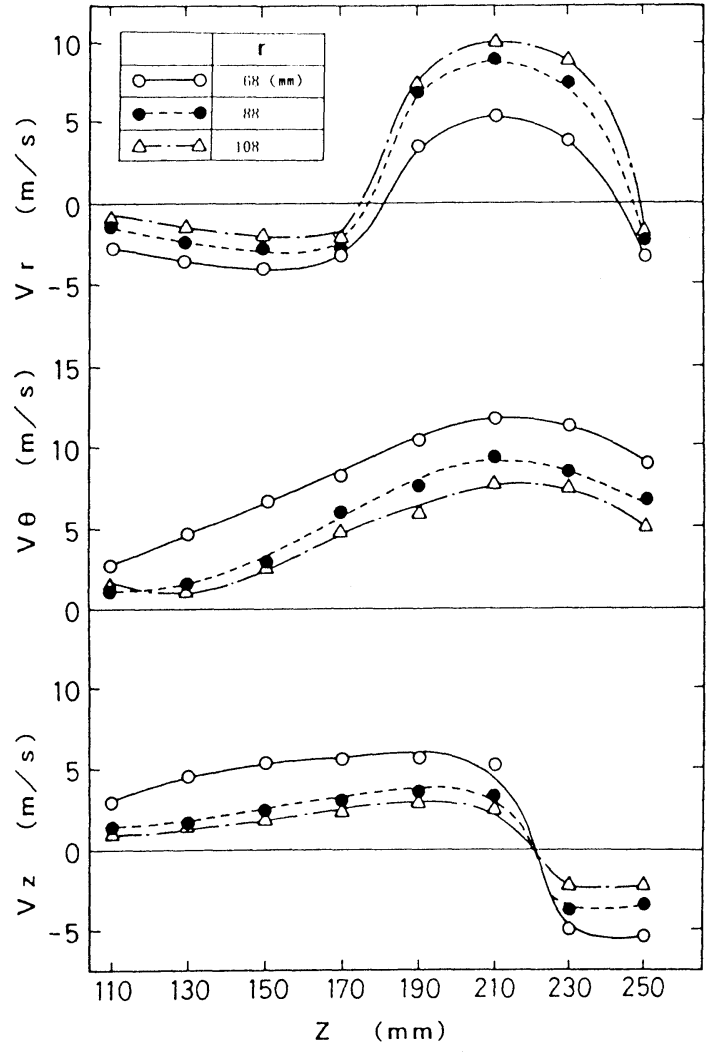

Fig. 4 Velocity of air flow around a rotating pirn (Nylon pirn 7,000 rpm)

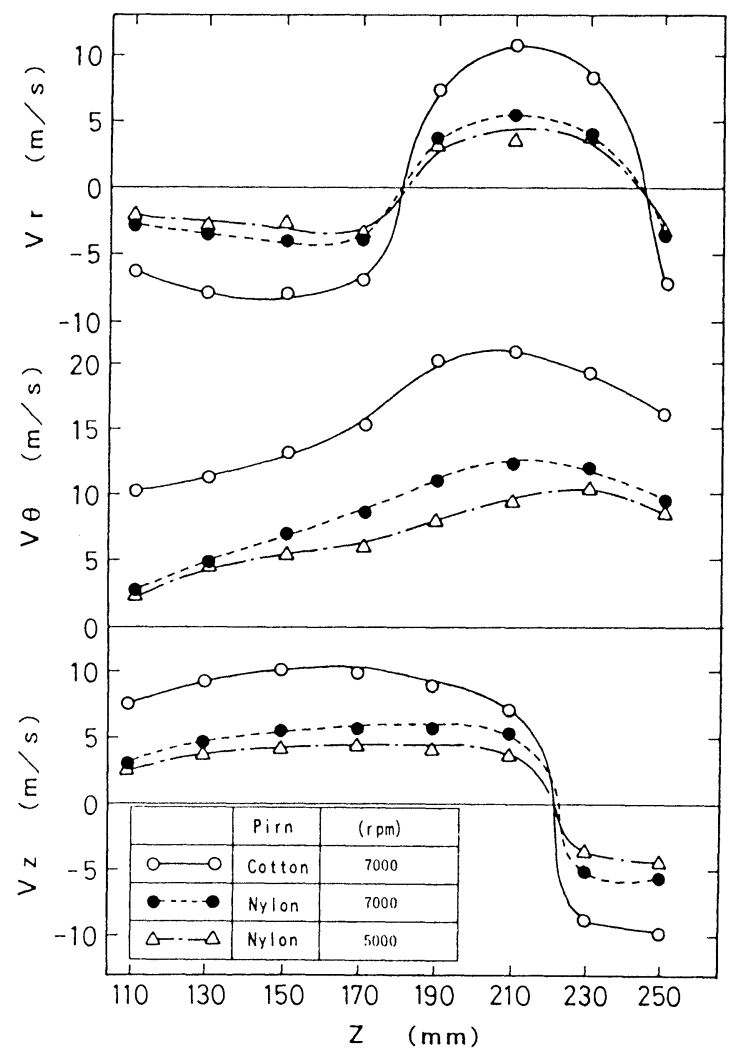

Fig. 5 Velocity of air flow around a rotating pirn $(r=68 \mathrm{~mm})$ revolutions, but have keen relation to the velocity magnitude as shown in Fig.5. The blow off width is about $1 / 3$ as long as the length of the cylindrical part, and the flow changes sharply its direction at the center of the pirn height. These phenomena have intimate relation to the energy consumption in rotating the pirn. To improve this flow, a cover as shown in Fig.6 was set around the rotating pirn.

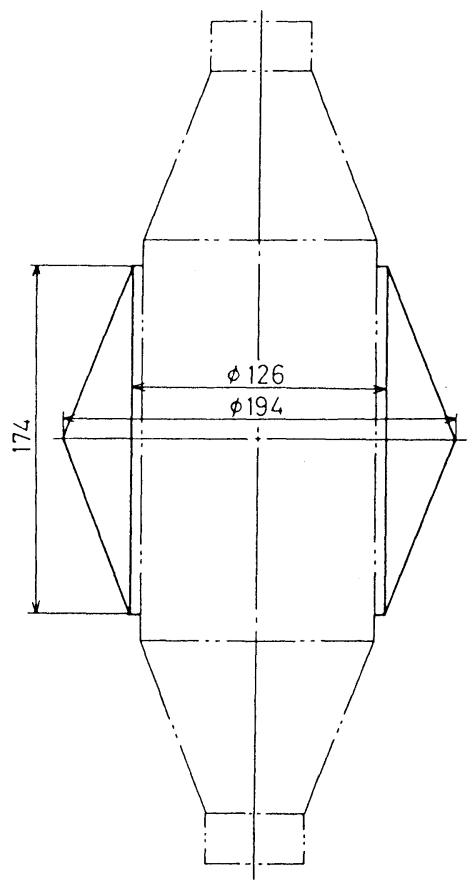

Fig. 6 Shape of cover

\section{Energy Consumption for Rotating a Pirn}

The reaction of the motor to rotate the pirn was measured by using an apparatus as shown in Fig.3. As a shaft projecting from both ends of the motor was supported by bearings, the motor case can rotate freely by the reaction around this shaft. This reaction was measured by the U-gauge and a strain amplifier by tying one end of a thread around the motor case and the other connected to the U-gauge. The power was represented by the product of the angular velocity $\omega_{m}$, the radius of the motor case and the net reaction force. Here the net reaction force is the force difference with and without a pirn. The revolution was changed from 2,000 to $7,000 \mathrm{rpm}$ at $200 \mathrm{rpm}$ interval. The power $L$ measured and the number of revolutions lead to the friction coefficient $C_{f}$ and Reynolds number $R e$ shown in Fig.7 by the following equation:

$$
L=C_{f} \cdot \rho \cdot V^{2} \cdot A_{p} / 2, \quad V=r_{p} \omega \ldots \ldots \ldots \ldots
$$

The Reynolds number is given as $r_{p}{ }^{2} \omega / \nu$ where $r_{p}$ is the maximum pirn radius, $\nu$ the kinematic viscosity of air, $\omega$ the angular velocity of the pirn. All curves in Fig.7 change their inclination at Reynolds number 0.9 


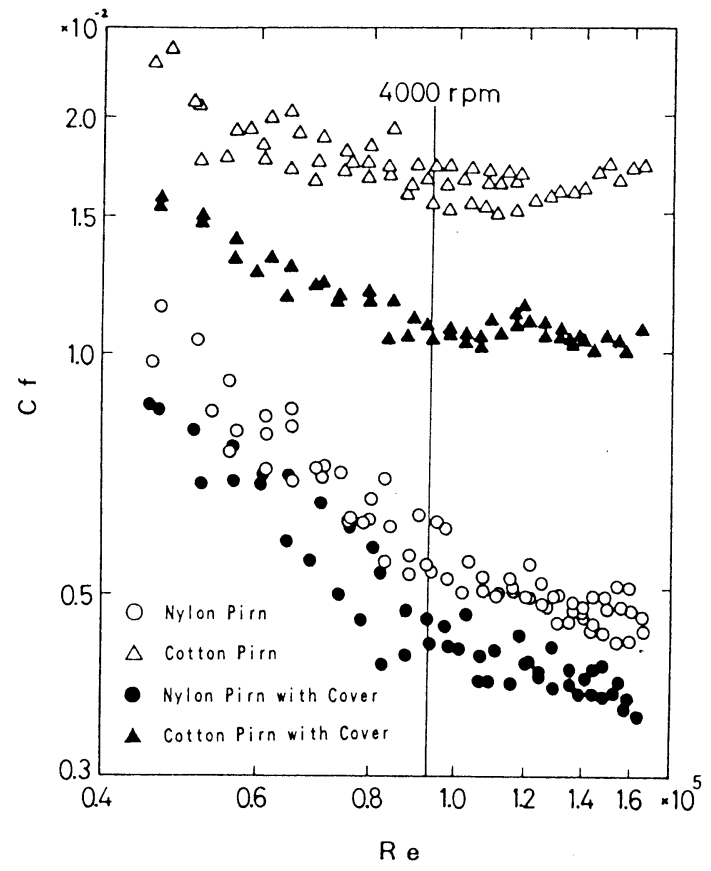

Fig. 7 Relation between friction coefficients $C_{f}$ and Reynolds number $R_{e}$

$\times 10^{5}$. Near this Reynolds number, the laminar boundary layer seems to become turbulent. In the case of the pirn with cotton yarns wound, its $C_{f}$ is about three times as large as that of a nylon pirn. This may be because the thickness of the turbulent boundary layer increases due to many vortices formed after fluffs.

When the cover was set around the the pirn, the energy consumption decreased about 20 and $40 \%$ for the nylon pirn and the cotton pirn, respectively. Therefore, the cover has significant effect on the energy consumption. In this case, the energy was consumed by shear stress inside the cover.

\section{Flow Analysis by a Finite Element Method}

\subsection{Procedure of calculation}

In order to analyse the air-flow around the rotating pirn, a finite element method was used on the assumption that the flow does not change its value in the circumferential component and air is incompressible. The cylindrical coordinate was used, and the air-flow was exhibited on the $r-\theta$ plane because the flow was regard as symmetrical with respect to the rotating axis.

The velocity was calculated by the continuity equation and Navier-Stokes equation. In order to solve these equations by a computer, stream function $\psi$ and vorticity $q$ were used[1] such as

$$
\begin{aligned}
& V_{r}=-\frac{1}{r} \frac{\partial \psi}{\partial Z}, V_{z}=\frac{1}{r} \frac{\partial \psi}{\partial r} \ldots \ldots \ldots \ldots \ldots \ldots \ldots \ldots \ldots \ldots \\
& q=\frac{\partial V_{r}}{\partial Z}-\frac{\partial V_{z}}{\partial r} \quad \ldots \ldots \ldots \ldots \ldots \ldots
\end{aligned}
$$

By rewriting the continuity equation and $V_{r}$ and $V_{z}$ in terms of $\psi, q$ and $V_{\theta}$, we have

$$
\begin{aligned}
& \frac{\partial^{2} \psi}{\partial Z^{2}}+\frac{\partial^{2} \psi}{\partial r^{2}}-\frac{1}{r} \frac{\partial \psi}{\partial r}=-r q \quad \ldots \ldots \\
& \frac{\partial q}{\partial t}+\frac{1}{r}\left(-\frac{\partial \psi}{\partial Z} \frac{\partial q}{\partial r}+\frac{\partial \psi}{\partial r} \frac{\partial q}{\partial Z}\right)+ \\
& \frac{q}{r^{2}} \frac{\partial \psi}{\partial Z}-\frac{1}{r} \frac{\partial\left(V_{\theta}^{2}\right)}{\partial Z}=\nu\left(\nabla q-\frac{q}{r^{2}}\right) \quad \ldots \\
& \frac{\partial V_{\theta}}{\partial t}+V_{r} \frac{\partial V_{\theta}}{\partial r}+V_{Z} \frac{\partial V_{\theta}}{\partial Z}+\frac{V_{r} V_{\theta}}{r}= \\
& \nu\left(\nabla V_{\theta}-\frac{V_{\theta}}{r^{2}}\right) \ldots \ldots \ldots \ldots \ldots
\end{aligned}
$$

In order to apply the finite element method, those $\psi, q, V_{\theta}$ are made discrete by the Gallerkin method. After the numerical calculation, the function $\psi, q$ are obtained and $V_{r}$ and $V_{z}$ are given by eq.(3).

The procedure to calculate $\psi, q$ and $V_{\theta}$ is shown in Fig.8. The integer $n$ there means the number of time step in calculation. At the first stage of calculation, the fluid field is thought as a potential flow. The calculation of $\psi . q$ and $V_{\theta}$ is progressed by increasing a slight time interval under the implicit method. Values of $q$ and $V_{\theta}$ on the boundary are decided by expanding $\psi, q$ and $V_{\theta}$ on the adjacent nodes into Taylor's series. Therefore, the

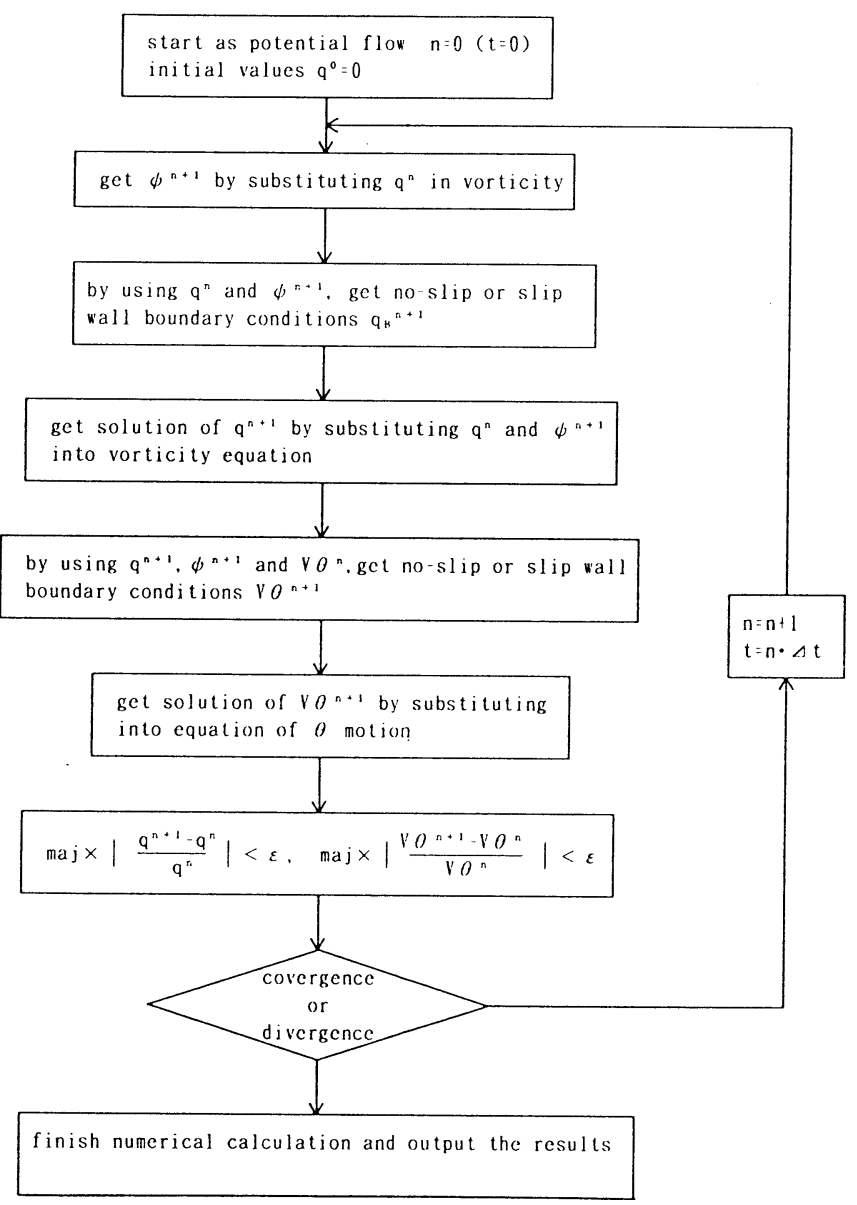

Fig. 8 Flowchart to analyse air flow 
boundary conditions is given in every time step. The calculation finishes when the velocity exceeds the maximum surface velocity of the pirn, or when the difference between each $q$ and $V_{\theta}$ for every interval becomes smaller than an arbitrary small value. That the velocity exceeds the maximum surface velocity is the case of the divergence and that the $q$ and $V_{\theta}$ remain constant for every step was convergence. In case of convergence, we can know the velocity in terms of the stream function and the vorticity.

The flow field to be analyzed must be divided into small regions called finite elements. The flow does not change in the region over $200 \mathrm{~mm}$ from the center, therefore the range $500 \mathrm{~mm} \times 180 \mathrm{~mm}$ is divided into trial elements consisting of 208 nodes and 348 elements as shown in Fig.9. When the cover is set around the pirn, the field is divided into 199 nodes and 316 trial elements as shown in Fig. 10. The boundary conditions on the pirn surface are the circumferential velocities shown as in Figs.10 and 11. The velocity at the cover is zero. Function $\psi$ equals 0 on the bottom face supporting the spindle and the pirn, where slip doesn't occur. In the case of the cotton pirn, the fluff resistance is taken into account. Namely, by using the fluff distributions in number and in length per $1 \mathrm{~m}$ of the yarn, the average

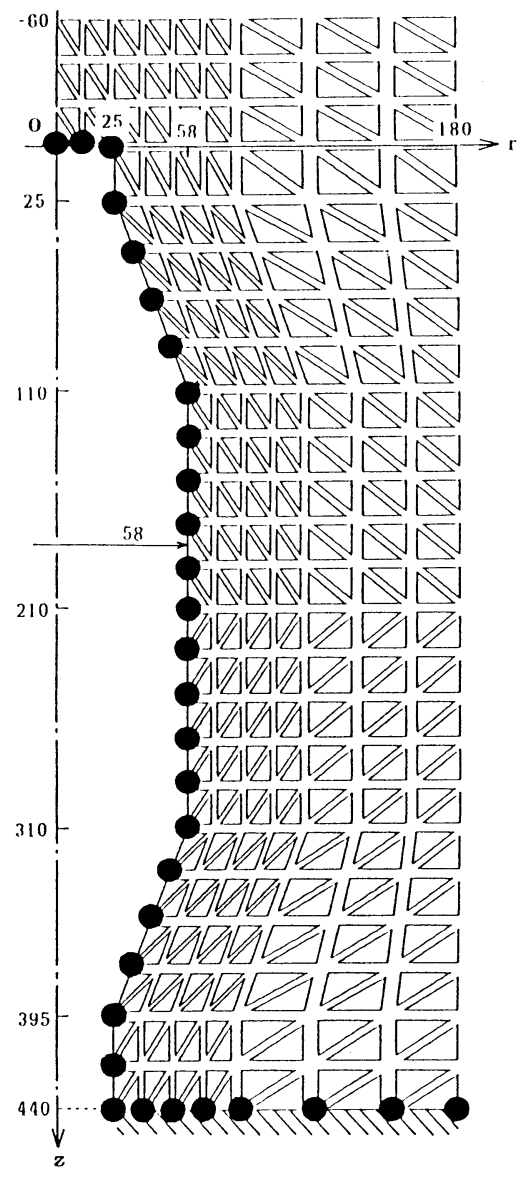

Fig. 9 Mesh data for analysis

(number of node: 208, number of element: 348 )

: boundary condition of $V_{\theta}$

IIII: no-slip wall

node of plane and pirn : $q=0$

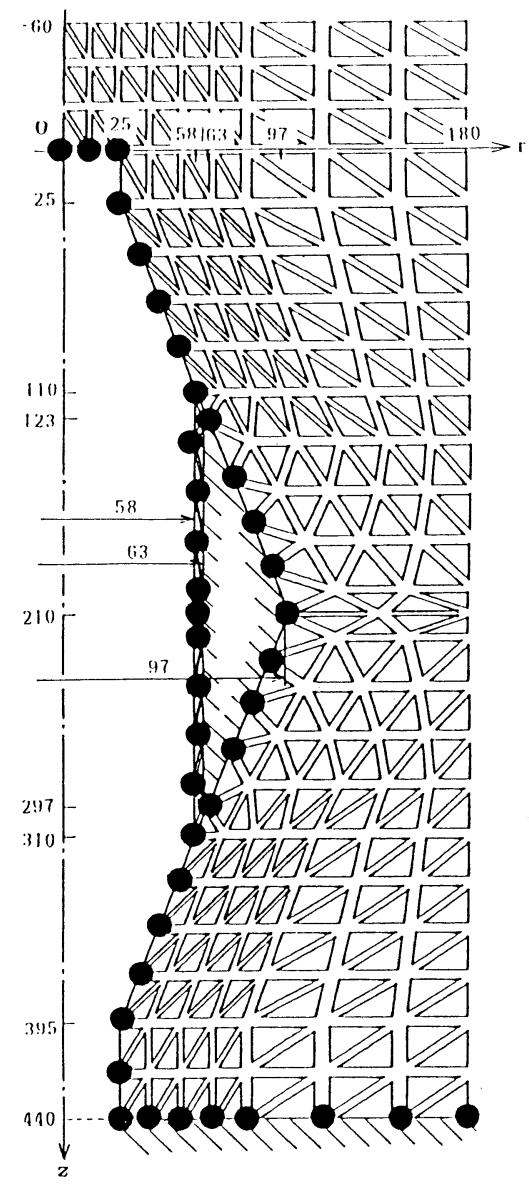

Fig. 10 Mesh data for analysis

(number of node: 199, number of element: 316 )

- : boundary condition of $V_{\theta}$

IIII : no-slip wall

node of plane and pirn : $q=0$

length of fluffs per unit area is estimated at $1 \mathrm{~mm}$ and their average number at 105 pieces. The fluff diameter is $0.01 \mathrm{~mm}$. In the calculation fluffs are replaced by slender bars projecting from the pirn and subjected to the air flow inducing drag-force.

\subsection{Result of analysis and velocity}

Velocities calculated with a computer are plotted in Fig.11, in which blow-off is seen all over the pirn, with particularly strong one in the vicinity of the center. To compensate this blow-off, suction is taken place at both ends of the pirn. But in experiment, suction is observed except the $6 \mathrm{~cm}$ range near the center. The circumferetial component has a minus sign when $Z$ is less than about $150 \mathrm{~mm}$. This means that the velocity is opposite to rotation. But this was not confirmed in the experiment and more research is required to verify the assumption by means of adequate sensors. The velocity along the axial direction increases slightly toward the center and changes the direction into reverse at the center. It was found that two flows sucked from the upper and the lower ends of the pirn strike against each other at the 


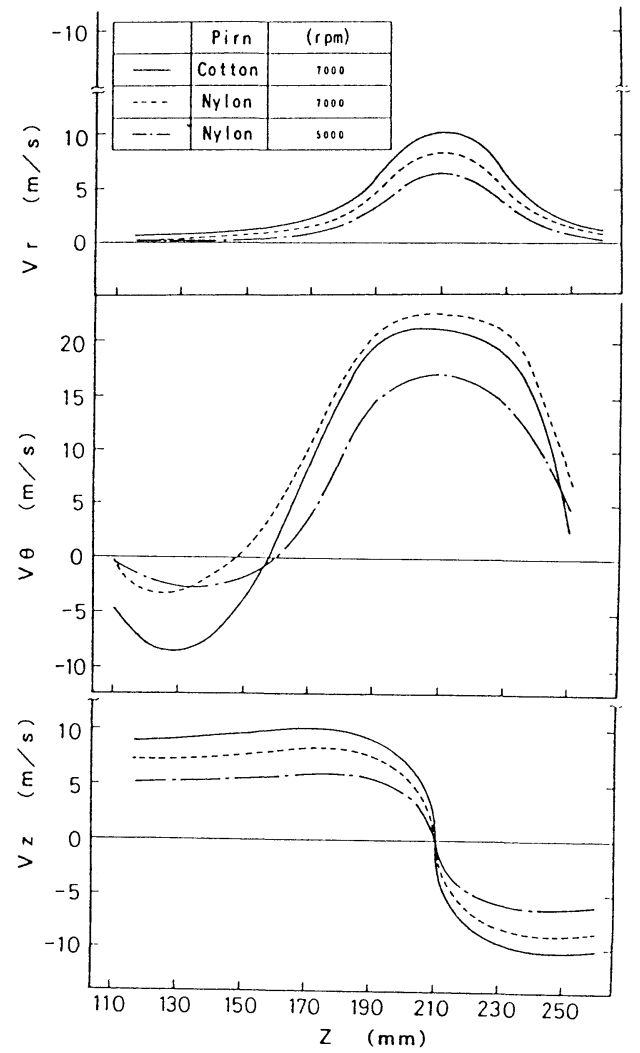

Fig. 11 Results of calculation

center and blow out outward. Thus, the improvement of this flow may reduce energy consumption.

Velocity vectors are represented on the $r-\theta$ plane in Figs.12 and 13. Left figures in these show results measured, and right are those of FEM calculations.

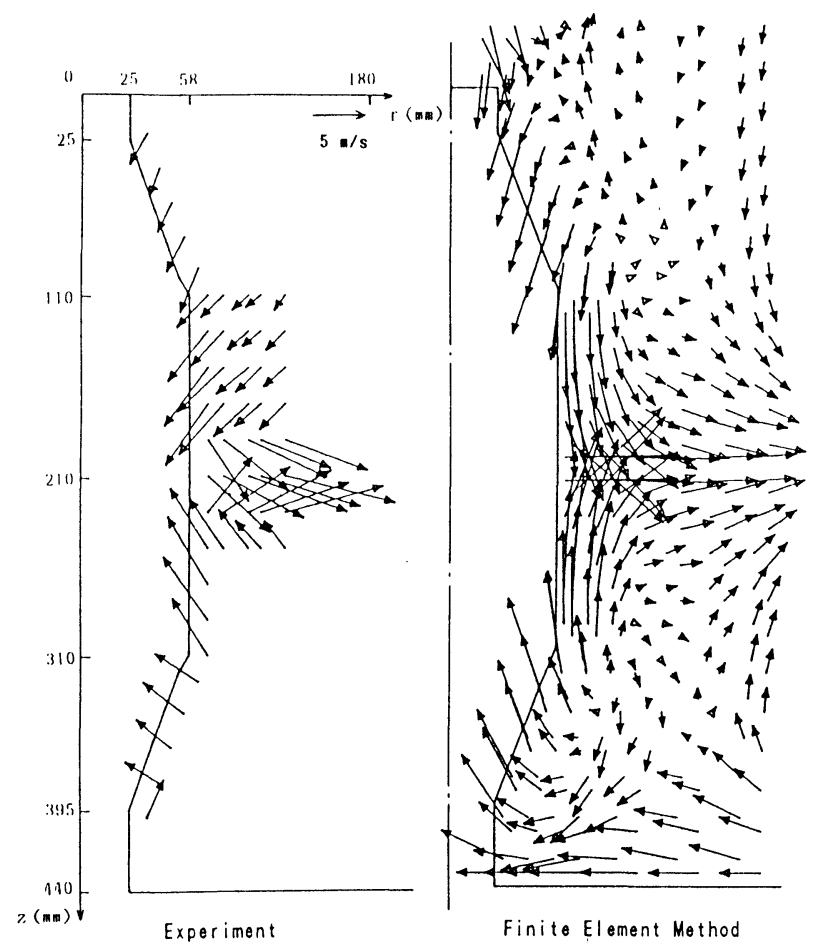

Fig. 12 Vectors around a pirn
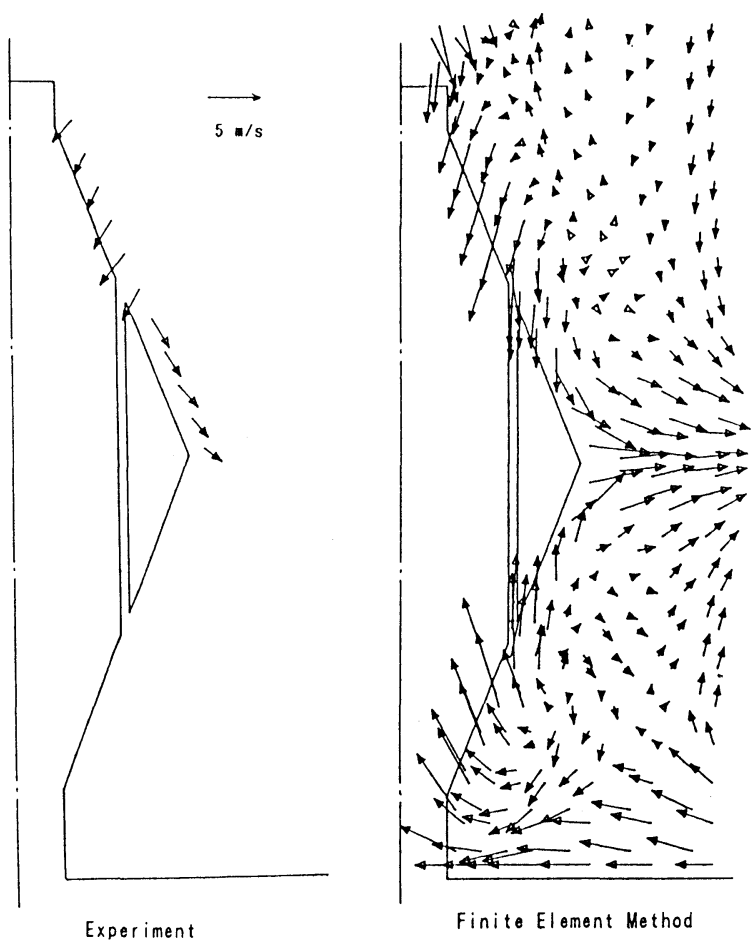

Fig. 13 Vectors around a pirn with a cover

\subsection{Estimation of energy consumption}

As air flow around the rotating pirn is similar to that of a pump, we can estimate the energy to rotate the pirn by means of both measured air velocity and calculated velocity, and compare it with measured power. The following procedure is employed in the calculation. Infinitesimal small air quantity $d Q$ is

$$
d Q=2 \pi r V_{r} d Z
$$

where $d Q$ is the rate of air volume flowing out from the imaginary cylindrical surface, and apart $10 \mathrm{~mm}$ from the pirn surface, having the height $d Z . V_{r}$ is the velocity blowing out from the surface $2 \pi r d Z$. The power $d L a$ of this fluid is represented as

$$
d L a=\rho d Q V^{2} / 2 \ldots \ldots \ldots \ldots \ldots
$$

Necessary power is the total of $d L a$ along the axis, and so.

$$
L_{a}=\pi \rho \Sigma r\left(V_{r}^{2}+V_{\theta}^{2}+V_{z}^{2}\right) \Delta Z
$$

In the case with the cover around the pirn, we consider the shear flow between the pirn surface and the cover. Suppose the pirn surface as a plane, the shearing force is calculated by the one-eighth power law and

$$
\tau=0.0296 \rho V_{s}^{2}\left(V_{s} \cdot l / \nu\right)^{-1 / 5}
$$

where $l$ is the peripheral length of the pirn, $V_{s}$ the pirn 
surface velocity. The total shear force is given by $\tau$ multiplied by the covered surface area $A_{p}$ of the pirn. Accordingly, the power is the product of the total shear force and the surface velocity, and

$$
L_{c}=\tau \cdot A_{p} \cdot V_{s}
$$

As shown in Table 1, agreement between power (1)

Table 1 Power measured, calculated and blown out

A B

$\mathrm{C}$

D

$\begin{array}{lllll}(1) & \text { Measured power } \quad 21.05 & 8.43 & 80.01 & 16.34\end{array}$

$\begin{array}{lllll}\text { (2) Calculated power } & 21.00 & 7.72 & 34.69 & 13.05\end{array}$

$\begin{array}{lllll}(3) & \text { Blown out power } \quad 12.95 & 6.05 & 60.65 & 12.24\end{array}$
A : Nylon 7,000 rpm
B : Nylon 5,000 rpm
C : Cotton 7,000 rpm
D : Nylon 7,000 rpm with
Cover

(1) Power obtained from motor rotation

(2) Power calculated by FEM

(3) Power calculated from blown out velocities obtained from motor rotation and power (2) calculated by FEM is good for smooth surface pirns, but power (2) calculated with the cotton surface is about 43 percent of power (1), and that with the cover is about 75 percent. In Table 1, power (3) means power calculated from blown out velocities. Each value of power (3) is about $62 \sim 75$ percent of power (1) according to the case.

\section{Conclusion}

The flow around a pirn rotating at high speed was measured, and analysed by using parameters such as the number of revolutions and pirn surface conditions. The results obtained are as follows.

1) It is shown quantitatively that air around a rotating pirn is sucked from the upper and the lower parts of the pirn and is blown out from the middle part.

2) When a cover is set around a rotating pirn, energy consumption is reduced about 20 percent.

3) The flow velocity was analysed by a finite element method, and the energy consumption calculated by this analysis agrees well with experiment for smooth surface pirns.

\section{Reference}

K. Hayashi et al.; "Flow Analysis by Personal computer", Asakurashyoten (1987) 